\title{
Percepción de la calidad del cuidado de Enfermería desde la perspectiva de personas hospitalizadas en una institución pública
}

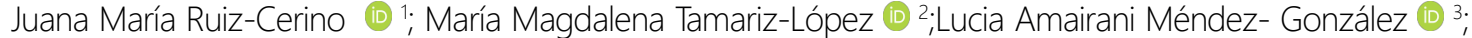 \\ Liliana Torres-Hernández (i] 4; Tirso Duran-Badillo 눙 5*
}

\section{RESUMEN}

Introducción: Los profesionales de Enfermería desde el ingreso al entorno hospitalario de una persona hasta el egreso, tienen constantemente un contacto directo, el evaluar cómo los pacientes perciben la calidad de la atención, permite desarrollar y establecer estrategias de mejora continua. Objetivo: Identificar la percepción de la calidad del cuidado de enfermería desde la perspectiva de personas hospitalizadas en una institución pública. Metodología: El estudio fue descriptivo, transversal, en una muestra compuesta por 90 pacientes mayores de 18 años identificados a través de un muestreo por conveniencia, se les aplicó el instrumento SERVQHOS-E, Alpha de Cronbach de .87. Resultados: En cuanto a la calidad del cuidado de enfermería, se encontró una media de 1.97 puntos ( $(\mathrm{EE}=.626)$ de satisfacción global. La mayoría de los pacientes indicaron estar satisfechos (67.8\%) con el cuidado de enfermería, seguida de muy satisfechos (18.9\%). Conclusiones: Más de la mitad de los sujetos que participaron en este estudio de manera general percibieron buena calidad del cuidado de enfermería.

Palabras clave: Percepción; Calidad de la atención de salud; Enfermería (DeCS)

1. Doctora en Metodología de la Enseñanza. Universidad Autónoma de Tamaulipas. Unidad Académica Multidisciplinaria Matamoros. Matamoros, Tamaulipas,México.

2. Licenciada en Enfermería. Universidad Autónoma de Tamaulipas. Unidad Académica Multidisciplinaria Matamoros. Matamoros, Tamaulipas, México.

3. Licenciada en Enfermería. Universidad Autónoma de Tamaulipas. Unidad Académica Multidisciplinaria Matamoros. Matamoros, Tamaulipas, México.

4. Licenciada en Enfermería. Universidad Autónoma de Tamaulipas. Unidad Académica Multidisciplinaria Matamoros. Matamoros, Tamaulipas, México.

5. Doctor en Metodología de la Enseñanza. Universidad Autónoma de Tamaulipas. Unidad Académica Multidisciplinaria Matamoros. Matamoros, Tamaulipas, México.

Recibido: $14 / 01 / 2020$

Aceptado: 22/06/2020

* Autor para correspondencia: tduran@docentes.uat.edu.mx

Copyright $\odot 2020$ SANUS

Artículo de acceso abierto distribuido

bajo Licencia Creative Commons

\section{cc) (i) $\Theta$

\section{Cómo citar este artículo}

Juana María Ruiz-Cerino JM, Tamariz-López MM, Méndez- González LA, Torres-Hernández L, Duran-Badillo. Percepción de la calidad del cuidado de Enfermería desde la perspectiva de personas hospitalizadas en una institución pública. SANUS. 2020; (14):1-9.[Acceso__ _ ]; Disponible en: 


\title{
Perception of the quality of nursing care from the perspective of people hospitalized in a public institution
}

\begin{abstract}
Introduction: From the moment a person enters a hospital until his discharge, nursing professionals constantly have a direct contact with the person; thus, evaluating how the patient perceived the quality of care he received allows the professionals to develop and implement strategies for continuous improvement. Objective: Identify the perception of the quality of nursing care from the perspective of people hospitalized in a public institution. Methodology: This was a cross-sectional descriptive study, using a sample comprised by 90 patients aged 18 and above who were identified through convenience sampling; the SERVQHOS-E instrument was applied with Cronbach's Alpha of .87. Results: Regarding the quality of nursing care, an average of 1.97 points ( $S D=.626$ ) of overall satisfaction was found. Most of patients indicated they were satisfied $(67.8 \%)$ with the nursing care, followed by very satisfied (18.9\%). Conclusions: More than half of the subjects who participated in this study, in a general manner perceived a good quality in the nursing care received.
\end{abstract}

Key words: Perception; Quality of health care; Nursing (DeCS). 


\title{
Percepção da qualidade da assistência de enfermagem na perspectiva de pessoas hospitalizadas em instituição pública
}

\begin{abstract}
ABSTRATO
Introdução: Os profissionais de enfermagem, desde a entrada no ambiente hospitalar da pessoa até a alta, mantêm contato direto constantemente, avaliando como os pacientes percebem a qualidade da assistência, permitindo desenvolver e estabelecer estratégias de melhoria contínua. Objetivo: Identificar a percepção da qualidade da assistência de enfermagem na perspectiva de pessoas hospitalizadas em uma instituição pública. Metodologia: $\bigcirc$ estudo foi descritivo e transversal. Em uma amostra composta por 90 pacientes com mais de 18 anos identificados por amostragem de conveniência, foi aplicado o instrumento SERVQHOS-E, Alpha da Cronbach da .87. Resultados: Em relação à qualidade da assistência de enfermagem, foi encontrada uma média de 1,97 pontos ( $D P=0,626)$ de satisfação geral. A maioria dos pacientes indicou estar satisfeita $(67,8 \%)$ com os cuidados de enfermagem, seguida de muito satisfeita (18,9\%). Conclusões: Mais da metade dos sujeitos que participaram deste estudo geralmente percebeu boa qualidade na assistência de enfermagem.
\end{abstract}

Palavras chave: Percepção; Qualidade dos cuidados de saúde; Enfermagem (DeCS). 


\section{INTRODUCCIÓN}

Las instituciones de salud a nivel mundial reconocen los desafíos que conlleva la llegada de un mundo globalizante, asumiendo la responsabilidad que tiene la calidad en la mejora continua de la atención (1). La calidad se define como las propiedades con las que cuenta un servicio para satisfacer las necesidades del cliente (2), en este sentido, la calidad del cuidado de enfermería puede entenderse como la forma en que los profesionales de enfermería implementan las intervenciones de manera oportuna y seguras para satisfacer las necesidades de los pacientes.

La perspectiva desde la que se puede valorar el máximo bienestar y seguridad del paciente es a través de la apreciación que el paciente posee sobre el cuidado que recibe durante su estancia hospitalaria, cuidado que se traduce en la satisfacción o insatisfacción del cliente/paciente (3). Las instituciones en los últimos años han tomado como estrategia la evaluación constante de la satisfacción, con el propósito de mejorar las percepciones que los pacientes tienen sobre la atención que les proporciona el profesional de Enfermería, que se traduce en un beneficio para la organización y funcionabilidad de los servicios ${ }^{(4)}$.

Además de la interacción del binomio enfermera paciente, se considera que la calidad en la atención debe ser reflejo del perfeccionamiento en los procesos de implementación de cuidados, situación que en múltiples ocasiones no se refleja, a pesar de la existencia y disponibilidad de los avances tecnológicos y el fácil acceso a la información, derivado del fenómeno de globalización que se vive actualmente, permitiendo la adqusición y fortalecimiento de conocimientos, así como el desarrollo de habilidades para el profesional de enfermería (5), a pesar de todos estos cambios sustanciales, continúan ocurriendo eventos adversos, que coadyuvan en ocasiones a elevar las tasas de morbilidad, costos, días de estancia hospitalaria y complicaciones patológicas, impactando significativamente en el paciente, familia y sociedad.

Para poder identificar y satisfacer oportunamente las necesidades de salud de los pacientes, el profesional de enfermería requiere de conocimientos y habilidades técnicas e interpersonales para brindar cuidados de calidad durante su hospitalización ${ }^{(6)}$. Debido a esto, se considera que es de gran importancia en la atención del paciente, ya que es el profesional de la salud que se encuentra en constante comunicación con la familia y paciente desde su ingreso, estancia hospitalaria, hasta el egreso; durante este lapso, el cuidado que otorgan los profesionales de enfermería conlleva cordialidad, empatía y atención individualizada, lo que repercute en la salud y bienestar del paciente ${ }^{(7)}$.

La calidad es un conjunto de acciones que van dirigidas hacia la excelencia en los servicios otorgados y por ende lograr la satisfacción del usarios ${ }^{\left({ }^{8}\right)}$. Enfermería es responsable de la calidad del cuidado que presta en una institución, además de la ética, leyes y normas de la profesión ${ }^{\left({ }^{9}\right)}$. Existe en la actualidad un interés en los hospitales por una búsqueda incesante de niveles más altos de calidad y de servicios, para ello se hace necesario desarrollar controles de calidad por las exigencias sociales. Se ha encontrado que, en las instituciones hospitalarias, las actuaciones de la enfermera son las más percibidas por los pacientes es decir que su actuación es interpretada más que de ningún otro participante como indicador de calidad por excelencia (10).

En un estudio realizado por Silva-Fhon y cols. ${ }^{(11)}$ se encontró una percepción del cuidado medianamente favorable con mayor alteración en los componentes técnico, interpersonal y confort. Mientras que en otro estudio realizado por Campiño-Valderrama y cols. ${ }^{(12)}$ los participantes indicaron percibir un cuidado humanizado.

Evaluar cómo percibe el paciente la calidad del cuidado de enfermería permite darse cuenta de cómo es la atención que se le brinda, además de que se pueden implementar diversas estrategias para mejorarla. Hoy en día la sociedad demanda un cuidado de calidad, para ello no solo es necesario que el profesional de enfermería domine los procedimientos que realiza sino debe ver al paciente holísticamente para poder satisfacer sus necesidades oportunamente. Por lo anterior, surge el siguiente objetivo de investigación: identificar la percepción de pacientes hospitalizados acerca de la calidad del cuidado de enfermería en una institución pública en Matamoros, Tamaulipas, México.

\section{METODOLOGÍA}

En esta investigación el diseño fue de tipo descriptivo y transversal. La población estuvo compuesta por pacientes hospitalizados en una institución pública de Matamoros, Tamaulipas, al noreste de México. El muestreo fue por conveniencia ya que se incluyó en el estudio a todos los pacientes hospitalizados en la institución anteriormente mencionada, en el momento de la recolección de datos. La muestra estuvo constituida por 90 pacientes mayores de edad. El criterio de inclusión que se consideró fue que los pacientes tuvieran la capacidad de comprender y responder las preguntas.

Para medir la calidad del cuidado se utilizó el instrumento SERVQHOS-E, está compuesto por 25 ítems, 12 evalúan la calidad subjetiva y 4 sobre calidad objetiva, calificados a través de una escala tipo Likert de 1 a 5 , es decir que oscila de 1 "mucho peor de lo que esperaba" a 5 "mucho mejor de lo que esperaba". En el segundo apartado del instrumento se encuentra una pregunta que indica la satisfacción global, posteriormente cuenta con 8 cuestionamientos relacionados con la percepción de la atención. Este instrumento ha sido utilizado y validado en población mexicana de pacientes con insuficiencia renal crónica y posee una confiabilidad de Alpha de Cronbach de 0.9113, en este estudio se encontró un Alpha de Cronbach de 0.87. Se apegó a los principios éticos estable- 
Tabla 1. Clasificación de la ocupación de los pacientes

\begin{tabular}{|l|c|c|}
\hline \multicolumn{1}{|c|}{ Ocupación } & $\boldsymbol{f}$ & \% \\
\hline Desempleado & 11 & 12.2 \\
\hline Empleado & 30 & 33.3 \\
\hline Actividades del hogar & 23 & 25.6 \\
\hline Trabajo independiente & 12 & 13.3 \\
\hline Pensionado & 9 & 10.0 \\
\hline Estudiante & 5 & 5.6 \\
\hline
\end{tabular}

Fuente: Datos Sociodemográficos

$\boldsymbol{n}=90$

\footnotetext{
$\mathrm{f}=$ frecuencia, $\%=$ porcentaje
}

cidos en la Declaración de Helsinki de la Asociación Médica Mundial (14)

De manera inicial se obtuvo la autorización del comité de ética e investigación de la Unidad Académica Multidisciplinaria Matamoros perteneciente a la Universidad Autónoma de Tamaulipas (Registro 051), además de la de los directivos de la institución pública de salud donde se realizó la investigación. Se abordó a los pacientes en su cama de hospitalización, inicialmente el investigador se presentó con cada paciente, posteriormente se les proporcionó el consentimiento informado, se aclararon dudas, se solicitó su firma y se les explicó que en caso de no participar no habría ninguna repercusión en la atención que se le estaba brindando, finalmente se aplicaron los instrumentos; una vez contestados, la información fue capturada en el programa estadístico Statistical Package for the Social Science (SPSS) versión 22 para Windows, donde se realizó estadística descriptiva, medidas de variabilidad (medias, desviación estándar) y medidas de posicionamiento (valor mínimo, valor máximo), para dar respuesta al objetivo planteado.

\section{RESULTADOS}

La muestra del presente estudio estuvo conformada por 90 pacientes hospitalizados de entre 18 y 75 años, de los cuales 53.3\% ( $f=48)$ correspondió a mujeres, la media de edad de 43.07 años $(D E=14.85)$. La escolaridad promedio fue de 8.61 años $(D E=4.15)$, solamente $3.3 \%(f=3)$ reportó no haber estudiado. El 54.4\% indicó no vivir con pareja marital. En la tabla 1 se observa la distribución de ocupación actual de los participantes.

El $67.8 \%$ de los participantes se encontraba hospitalizado en el servicio de cirugía, $14.4 \%$ en medicina interna, $7.8 \%$ en traumatología y $10.0 \%$ en ginecoobstetricia. Respecto a la satisfacción global de la calidad del cuidado de enfermería, en una escala de 1 a 4 puntos, se encontró una media de 1.97 puntos $(D E=0.626)$. La mayoría de los pacientes indicaron estar satisfechos con el cuidado de enfermería, seguido de muy satisfechos (Tabla 2).

Respecto a la calidad objetiva, en la tecnología de equipos la mayoría indicó que la calidad fue como se lo esperaba. Sobre la apariencia del personal de enfermería, señalizaciones del hospital y estado de las habitaciones la mayoría indicaron que fue mejor de lo que se esperaba (Tabla 3). La calidad subjetiva en todas las variables el mayor porcentaje de los pacientes indicaron que fue mejor de lo que se esperaban (Tabla 4).

Finalmente la mayoría de los pacientes indicó que sin dudarlo recomendaría el hospital, la mayoría reportó que le pidieron su consentimiento para realizarle pruebas o procedimientos, y que el tiempo de hospitalización fue el necesario, solamente un poco más de la mitad conoce el nombre de la enfermera, la mayoría indicó que la información que ha recibido es suficiente, todos reportaron conocer el servicio en el que se encuentran hospitalizados, la mayoría ingresó por urgencias y había sido hospitalizado dos o más veces (Tabla 5).

\section{DISCUSIÓN}

En este estudio se constató empíricamente que en la percepción de la calidad del cuidado de enfermería aproximadamente dos tercios de los participantes estuvieron satisfechos y una quinta parte refirió estar muy satisfechos con los cuidados y atención general que reciben por parte de los profesionales de enfermería, estos hallazgos son similares a los reportados por otros autores (3); lo que se traduce en una importante acción de los profesionales de enfermería con impacto en el desempeño y logro de metas institucionales, ya que refleja la calidad asistencial. Por otra parte, contribuye en el mejoramiento del paciente, directa e indirectamente a través de las intervenciones y motivación continua para su autocuidado.

Respecto a la calidad objetiva, en la variable de tec- 
Tabla 2. Clasificación de la percepción de la satisfacción global del cuidado de enfermería

\begin{tabular}{|l|c|c|}
\hline \multicolumn{1}{|c|}{ Satisfacción } & $\boldsymbol{f}$ & \% \\
\hline Muy satisfecho & 17 & 18.9 \\
\hline Satisfecho & 61 & 67.8 \\
\hline Poco satisfecho & 10 & 11.1 \\
\hline Nada satisfecho & 2 & 2.2 \\
\hline
\end{tabular}

Fuente: SERVQHOS-E

$\boldsymbol{n}=90$

$f=$ frecuencia, \%= porcentaje

Tabla 3. Percepción de los pacientes de la calidad objetiva del cuidado de enfermería

\begin{tabular}{|l|c|c|c|c|c|c|c|}
\hline \multirow{2}{*}{\multicolumn{1}{c|}{ Variable }} & \multicolumn{5}{c|}{ Escala \% (f) } & \multirow{2}{*}{ Media } & \multirow{2}{*}{ DE } \\
\cline { 2 - 8 } & $\mathbf{1}$ & $\mathbf{2}$ & $\mathbf{3}$ & $\mathbf{4}$ & $\mathbf{5}$ & & \\
\hline La tecnología de equipos & $0(0)$ & $7.8(7)$ & $63.3(57)$ & $24.4(22)$ & $4.4(4)$ & 3.26 & .663 \\
\hline La apariencia personal enfermería & $1.1(1)$ & $7.8(7)$ & $25.6(23)$ & $53.3(48)$ & $12.2(11)$ & 3.68 & .832 \\
\hline Señalizaciones del hospital & $1.1(1)$ & $5.6(5)$ & $33.3(30)$ & $50(45)$ & $10(9)$ & 3.62 & .787 \\
\hline Estado habitaciones & $0(0)$ & $4.4(4)$ & $37.8(34)$ & $40(36)$ & $17.8(16)$ & 3.71 & .811 \\
\hline
\end{tabular}

Fuente: SERVQHOS-E

1= Mucho peor de lo que esperaba, 2= Peor de lo que esperaba, 3= Como me lo esperaba, 4= Mejor de lo que me esperaba,

$5=$ Mucho mejor de lo que me esperaba

nología de equipos, la mayoría indicó que la calidad fue como se lo esperaba y en las variables de apariencia del personal de enfermería, señalizaciones del hospital y el estado de las habitaciones, la mayoría indicaron que fue mejor de lo que se esperaba, similar a lo reportado por Barragán y Moreno (15). En cuanto a la calidad subjetiva del servicio de enfermería, en todas las variables un mayor porcentaje de pacientes indicaron que la calidad de la atención fue mejor de lo que se esperaban, este dato es congruente con lo encontrado por otros autores ${ }^{(15,16)}$. Lo que significa que en la actualidad tanto los integrantes de los sistemas administrativos institucionales como los gestores del cuidado conocen la importancia de disponer de áreas centradas en las necesidades de los usuarios, sobre todo en el otorgamiento del cuidado más humano.

La mayoría de los usuarios indicó que sin dudarlo recomendaría el hospital, la mayoría reportó que le pidieron su consentimiento para realizarle procedimientos, el período de hospitalización fue el necesario, la comunicación fue adecuada, la mayoría ingresó por urgencias y había sido hospitalizado dos o más veces; estos resultados coinciden con lo encontrado por otros autores $(15,17)$.

En el presente estudio se encontró que solamente un poco más de la mitad conoce el nombre de la enfermera, similar a lo reportado en otro estudio ${ }^{(17)}$ y todos reportaron conocer el servicio en el que se encuentran hospitalizados, diferente de lo reportado por Borré y Vega ${ }^{(17)}$. Lo que indica que los profesionales de enfermería constantemente están modificando estilos y estrategias de intervención en bien del paciente y centrado en las necesidades individuales.

Con los hallazgos de este estudio es evidente lo que los profesionales de Enfermería constituyen un elemento de gran importancia para la calidad de la atención del paciente hospitalizado que va más allá de la ejecución correcta de los cuidados sino de una atención integral.

\section{CONCLUSIONES}

De acuerdo con el objetivo planteado para el presente estudio, el cual fue identificar la percepción de pacientes hospi- 
Tabla 4. Percepción de los pacientes de la calidad subjetiva del cuidado de enfermería

\begin{tabular}{|c|c|c|c|c|c|c|c|}
\hline \multirow{2}{*}{ Variable } & \multicolumn{5}{|c|}{ Escala \% (f) } & \multirow{2}{*}{ Media } & \multirow{2}{*}{$D E$} \\
\hline & 1 & 2 & 3 & 4 & 5 & & \\
\hline Interés por cumplir lo que promete & $0(0)$ & $1.1(1)$ & $36.7(33)$ & $43.3(39)$ & 18.9(17) & 3.80 & .753 \\
\hline Información & $0(0)$ & $5.6(5)$ & $28.9(26)$ & $50(45)$ & $15.6(14)$ & 3.76 & .78 \\
\hline Tiempo de espera & $0(0)$ & $6.7(6)$ & $32.2(29)$ & $47.8(43)$ & $13.3(12)$ & 3.68 & .791 \\
\hline Interés por solucionar & $0(0)$ & $6.7(6)$ & 25.6(23) & $51.1(46)$ & $16.7(15)$ & 3.78 & .804 \\
\hline Puntualidad & $0(0)$ & 1.1(1) & 31.1(28) & $44.4(40)$ & 23.3(21) & 3.90 & .765 \\
\hline Rapidez & $0(0)$ & 1.1(1) & $32.2(29)$ & $44.4(40)$ & $22.2(20)$ & 3.88 & .762 \\
\hline Disposición & $0(0)$ & 1.1(1) & 18.9(17) & $53.3(48)$ & $26.7(24)$ & 4.06 & .709 \\
\hline Confianza & $0(0)$ & $2.2(2)$ & $26.7(24)$ & $41.1(37)$ & $30(27)$ & 3.99 & .814 \\
\hline Amabilidad & $0(0)$ & $6.7(6)$ & 21.1(19) & $48.9(44)$ & 23.3(21) & 3.89 & .841 \\
\hline Preparación & $0(0)$ & $7.8(7)$ & $22.2(20)$ & $42.2(38)$ & $27.8(25)$ & 3.90 & .900 \\
\hline Trato personalizado & $0(0)$ & $5.6(5)$ & $22.2(20)$ & $37.8(34)$ & $34.4(31)$ & 4.01 & .893 \\
\hline Comprensión de necesidades & 1.1(1) & $5.6(5)$ & $16.7(15)$ & $38.9(35)$ & $37.8(34)$ & 4.07 & .934 \\
\hline
\end{tabular}

Fuente: SERVQHOS-E

1= Mucho peor de lo que esperaba, 2= Peor de lo que esperaba, 3=Como me lo esperaba, 4= Mejor de lo que me esperaba,

$5=$ Mucho mejor de lo que me esperaba

talizados acerca de la calidad del cuidado de enfermería en una institución pública, se encontró que aproximadamente dos tercios de los participantes indicaron estar satisfechos y una quinta parte refirió estar muy satisfechos. En cuanto a los cuestionamientos acerca de la apariencia o imagen de la enfermera, solamente la mitad refirió estar satisfecho y menos de la mitad está satisfecho con la confianza que el profesional proyecta. Por los resultados encontrados se recomienda que se sigan realizando investigaciones que aborden las causas, ya que esto permite justificar el diseño de acciones encaminadas a redireccionar la calidad del cuidado, mediante estrategias de intervención desde la formación, el ejercicio, la asociación profesional que permite sensibilizar al personal de enfermería sobre la necesidad de ofrecer cuidado de excelencia que contribuya a la mejora de la práctica y la imagen.

Entre las limitaciones del presente estudio se consideró el muestreo no probabilístico y tamaño de muestra, por lo que es necesario tomar con cautela estos resultados; no obstante, se considera un importante avance a la comprensión de la calidad del cuidado de enfermería.

\section{CONFLICTO DE INTERESES}

Los autores declaran no tener conflicto de intereses.

\section{FINANCIAMIENTO}

Proyecto sin financiamiento.

\section{REFERENCIAS BIBLIOGRÁFICAS}

1. Robledo HG, Fajardo G, García S. Reflexiones en torno al hexágono de calidad de los servicios de salud. En la calidad de la atención a la salud en México a través de sus instituciones ( $2^{\mathrm{a}}$ ed.). Biblioteca Mexicana del conocimiento [Internet]. 2015, [consultado Mayo 2020]; p. 47-85. Disponible en: https://www.gob.mx/cms/uploads/attachment/file/60110/libro_03.pdf

2. Llinás DA. Evaluación de la calidad de la atención en salud, un primer paso para la reforma del sistema. Salud Uninorte [Internet]. 2010; [Consultado Marzo 2019]; 26(1): 143-54. Disponible en: http://www.scielo.org.co/scielo. php?script=sci_arttext\&pid $=$ S0120 $-55522010000100014 \& \mid-$ $\mathrm{ng}=\mathrm{en}$

3. Lenis-Victoria CA, Manrique-Abril F. Calidad del cuidado de enfermería percibida por pacientes hospitalizados en 
Tabla 5. Percepción de los pacientes de la satisfacción de calidad del cuidado de enfermería

\begin{tabular}{|l|l|c|c|}
\hline \multicolumn{1}{|c|}{ Variable } & \multicolumn{1}{|c|}{ Escala } & $\boldsymbol{f}$ & $\%$ \\
\hline \multirow{2}{*}{ Recomendaría el hospital } & Sin dudarlo & 62 & 68.9 \\
& Tengo dudas & 21 & 23.3 \\
& Nunca & 7 & 7.8 \\
\hline Consentimiento de pruebas o procedimientos & Si & 50 & 55.6 \\
& No & 24 & 26.6 \\
\hline & Algunas veces & 16 & 17.8 \\
\hline Tiempo de hospitalización & Menos de lo necesario & 7 & 7.8 \\
& El tiempo necesario & 69 & 76.7 \\
\hline Conoce el nombre de la enfermera & Más de lo necesario & 14 & 15.6 \\
\hline Información suficiente & Si & 55 & 61.1 \\
& No & 35 & 38.9 \\
\hline Conoce el servicio donde está hospitalizado & Si & 62 & 68.9 \\
\hline Ingreso al hospital & No & 28 & 31.1 \\
\hline Húmero de & Reporta & 90 & 100 \\
\hline & No reporta & 0 & 0 \\
\hline & Programado & 30 & 33.3 \\
& Por urgencias & 51.1 \\
\hline
\end{tabular}

Fuente: SERVQHOS-E

una clínica privada de Barranquilla (Colombia). Aquichan [Internet]. 2015; [Consultado Marzo 2019]; 15(3): 413-425. Disponible en: http://dx.doi.org/10.5294/aqui.215.15.3.9

4. Lyu H, Wick EC, Housman M, Freischlag JA, Makary MA. Patient satisfaction as a possible indicator of quality surgical care. JAMA Surg [Internet]. 2013 [consultado Marzo 2019]; 148(4): 362-7. Disponible en: http://dx.doi. org/10.1001/2013.jamasurg.270

5. Monje P, Miranda P, Oyarzün J, Seguel F, Flores E. Percepción de cuidado humanizado de enfermería desde la perspectiva de usuarios hospitalizados. Cienc. Enferm. [Internet]. 2018 [consultado Mayo 2019]; 24(5): 1-10. Disponible en: http://dx.doi.org/10.4067/s0717-95532018000100205
6. Puch-Ku GF, Uicab-Pool GA, Ruiz-Rodríguez M, Castañeda-Hidalgo H. Dimensiones del cuidado de enfermería y la satisfacción del paciente adulto hospitalizado. Rev Enferm Inst Mex Seguro Soc [Internet]. 2016 [consultado Marzo 2019]; 24(2): 123-8. Disponible en: https://biblat.unam.mx/hevila/RevistadeenfermeriadelInstitutoMexicanodelSeguroSocial/2016/ vol24/no2/8.pdf

7. Blasco-León M, Ortíz-Luis SR. Ética y valores en Enfermería. Rev Enferm Inst Mex Seguro Soc [Internet]. 2016 [consultado Mayo 2020]; 24(2): 145-9. Disponible en: https://www.medigraphic.com/pdfs/enfermeriaimss/eim-2016/eim162l.pdf

8. Febré N, Mondaca-Gómez K, Méndez-Celis P, Badilla-Morales V, Soto-Parada P, Ivanovic P, Reynaldos K, Canales M. Calidad en Enfermería: su gestión, implementación y medición. 
Rev. Med. Clin. Condes. [Internet]. 2018 [consultado Mayo 2020]; 29(3): 278-87. Disponible en: https://doi.org/10.1016/j. rmclc.2018.04.008

9. Escobar-Castellanos B, Cid-Henríquez P. El cuidado de enfermería y la ética derivados del avance tecnológico en salud. Acta Bioet. [Internet]. 2018 [consultado Mayo 2020]; 24(1): 39-46. Disponible en: https://scielo.conicyt.cl/pdf/abioeth/ v24n1/1726-569X-abioeth-24-01-00039.pdf

10. Chávez D, Romeo R, Zúñiga J. Percepción de la calidad del Cuidado de Enfermería en Pacientes en el hospital Universitario del Caribe, Cartagena. (Tesis de licenciatura). Universidad de Cartagena, Cartagena de Indias; 2013.

11. Silva-Fhon J, Ramón-Cordova S, Vergara-Villanueva S, Palacios-Fhon, Partezani-Rodrigues R. Percepción del paciente hospitalizado respecto a la atención de enfermería en un hospital público. Enfermería Universitaria. [Internet]. 2015 [consultado Mayo 2020]; 12(2):80-87. Disponible en: http://dx.doi. org/10.1016/j.reu.2015.04.001

12. Campiño-Valderrama SM, Duque PA, Cardozo VH. Percepción del paciente hospitalizado sobre el cuidado brindado por estudiantes de enfermería. Univ. Salud. [Internet]. 2019 [consultado Mayo 2020]; 21(3):215-25. Disponible en: http://dx.doi. org/10.22267/rus.192103.158

13. Castro-Serralde E, Cadena-Antonio G. Dimensiones de la satisfacción del paciente con insuficiencia renal crónica respecto a la calidad del cuidado de enfermería. Rev Enferm Inst Mex Seguro Soc. [Internet]. 2017 [consultado Marzo 2019]; 25(4):271-8. Disponible en: http://revistaenfermeria. imss.gob.mx/editorial/index.php/revista_enfermeria/article/ view/246/859

14. De Almeida $T$, Aparecida $Y$, Pires $D$, Lebrão ML, Satya $M$, Dos Santos A, Amaro E. Factors associated with lower gait speed among the elderly living in a developing country: a crosssectional population-based study. BMC Geriatr [Internet]. 2015 [consultado Marzo 2019]; 15(35): 1-9. Disponible en: https://doi.org/10.1186/s12877-015-0031-2

15. Barragán JA, Moreno CM. Calidad percibida por usuarios de enfermería en tres hospitales públicos. Enfermería Global [Internet]. 2013; [consultado Marzo 2019];12(29): 217-230. Disponible en: http://scielo.isciii.es/scielo.php?script=sci_arttext\&pid=S1695-61412013000100011\&lng=es

16. Martin RA. Satisfacción del paciente en hemodiálisis como criterio de calidad asistencial en enfermería. Revista Mexicana de Urología [Internet]. 2014 [consultado en Marzo 2019]; 74(5): 292-5. Disponible en: https://doi.org/10.1016/j. uromx.2014.09.008
17. Borré YM, Vega Y. Calidad percibida de la atención de enfermería por pacientes hospitalizados. Ciencia y enfermería [Internet]. 2014 [consultado en Marzo 2019]; 20(3): 81-94. Disponible en: https://doi.org/10.4067/S071795532014000300008 